\title{
Research on the Pressure Comfort of Sports Bra
}

\author{
Wang Erhui, Wang Yanzhen* \\ Fashion Institute, Shanghai University of Engineering Science, Shanghai, China \\ Email address: \\ 18317135561@163.com (Wang Erhui),wangyanzhen1227@126.com (Wang Yanzhen) \\ ${ }^{*}$ Corresponding author
}

\section{To cite this article:}

Wang Erhui, Wang Yanzhen. Research on the Pressure Comfort of Sports Bra. Science Research. Vol. 5, No. 1, 2017 , pp. 6-9. doi: 10.11648/j.sr.20170501.12

Received: January 13, 2017; Accepted: February 6, 2017; Published: March 11, 2017

\begin{abstract}
The pressure comfort of sports bra mean women can meet the requirement of comfort in pressure of corsage when doing sports. In this paper, based on three different exercise intensity, discussed the related research on pressure comfort of sports bar. For women in the movement of different project, choosing sports bras to provide the reference.
\end{abstract}

Keywords: Sports Bra, Pressure Comfort, Exercise Intensity

\section{Introduction}

With the improvement of people's health awareness, domestic set off an upsurge of fitness in recent years. All sorts of sports are highly welcome. Sports bras are associated with movement of the demand arises at the historic moment, designed to meet the demand of women's movement at the same time have very good protection effect to the breast. For women, it is necessary to wear sports bras When doing sports [14]. The pressure comfort of sports bra is about efficiency of the movement and breast health, has become a research hotspot.

\section{The Generation of Sports Bra Pressure}

The pressure of the corset refers to the factors of bra size, weight, shape produce pressure when restrict the breast after women dressing. Bra pressure mainly includes two aspects to the human body. One is from the weight of the corset. When the shoulder belt near the shoulder point pressure value is bigger, it has to do with clothing in the longitudinal and the human body near the shoulder point of contact. The contact position is affected by design, material and size [15]. The other is resulting from Human silhouette and human movement form a dynamic contact between the clothing and body parts. In the process of human body dynamic contact with clothing, clothing and relative slippage and deformation fabrics to cope with change. Due to the deformation of fabrics can produce internal stress (Including the tensile, compression, shear and bending stress), So it can produce force binding to human body contact parts, cause skin deformation and stimulate skin deep pressure point makes the wearer feel the clothing pressure.

If the bra pressure is too great, the human body movement in order to overcome this resistance is bound to make a lot of lost work, so as to make people feel tired. Seriously will affect the body's breath, pulse and blood circulation, detrimental to human's physical and mental health; Proper clothing pressure will produce certain positive role to the human body. People when doing sports, wearing pressure appropriate sports bras will enhance the efficiency of the movement of athletes and increase their ability of tolerance. So the clothing pressure comfort is extremely important for human health.

\section{The Division of Sports Bras and Exercise Intensity}

With the development of science and technology, the mature human ergonomics and the world of competitive sport extensively developed, The pressure comfort Sports bras for female athletes and sports fans is very important. General sports bras than ordinary corset short, good elasticity, body-fitted and its function is when the human body movement, the human body skin, bone and muscle stretching 
and contraction, protect the breast, breast support, control the displacement of the breast to avoid breast muscle and ligament damage, reduce the female chest pain [12]. On the premise of not impede movement, it has some functions include protection, stability, moisture absorption and sweat. If you don't wear sports bras, chest involuntarily swing sharply, especially the chest type larger women, make the elastic fiber inside the breast tissue is permanent damage, resulting in bosom prolapse.

Now researchers are mostly pay attention to the study of bra comfort, especially in the pressure comfort of bra breast research is more outstanding [13]. Most of the research is by asking subjects to wear a particular sport bra, remain at rest or doing a particular action, recorded in pressure gauge pressure value at the same time or let the experimenter evaluation of the clothing pressure subjective and wearing comfort of human body. Related research found that the clothing pressure with human subjective evaluation of clothing comfort is of certain linear relationship, and wearing a sports bra, human body each part of stress are different. The ideal sports bra pressure is within the scope of the human body can withstand because the appropriate pressure can reduce muscle vibration and unnecessary energy loss and can massage, and enhance the contraction of muscle force, at the same time with a good fashion model [11]. In order to solve these problems, researchers and manufacturers around the world started to research and development of sports bra, and do a lot of related research.

According to different types of movement at home and abroad, studied the pressure comfort of sports bra. Different sports have different exercise intensity. Different sports have different exercise intensity, so the chest of force and vibration amplitude is also different in different sports. Sports according to the division strength, can be divided into three categories:

(1) low intensity exercise: walking, yoga, playing golf, tai-ji, swimming

(2) medium intensity exercise: skiing, walking race, go boating, skating, playing tennis, jogging, Aerobic pedal

(3) high intensity exercise: playing basketball, gymnastics, running, playing football, playing volleyball, High impact aerobic dancing

Through the division of exercise intensity, grasps the women in the chest of the margin of shaking when motion for the research of sports bra pressure comfort provides a new way of thinking.

\section{The Corset under Different Intensity of Pressure Comfort}

\subsection{Low Intensity Exercise}

In order to study the same body size of women wearing the same bra styles in different positions, the pressure between the body size In the case of comfortable. Xiao-Jun Ding used the AMI - 3037 airbag clothing pressure tester of Japan AMI THE CHNO company and experiment. Select 20 representative of women, and a specific shape of the bra. Objective pressure value is measured under different condition first, Then according to the subjective evaluation, it is concluded that to design clothing should be in accordance with the size of each part of the human body the pressure and meet the clothing wearing comfortable [1]. This shows that Exercise chest pressure parts size is all different In the stationary state and within a certain range of pressure value, the human body feeling is comfortable. Athletes at a low intensity exercise like tai ji, walking etc. You can refer to the pressure comfort value Under this condition, choosing the right sports bra.

Yu Shi jia through the study of pressure comfort of women's seamless underwear, to improve the pressure comfort of seamless underwear. It is concluded that under different motion state of clothing pressure comfortable scope. He used an improved method of Fuzzy closeness to solve the weight on the site of each pressure test point, and the test point pressure value can be converted to the parts of pressure value. Using the method of parameter interval estimation for comfortable value carried out a statistical analysis on the pressure value, get each part under different motion state of comfortable clothing pressure range [2]. By combining subjective and objective evaluation of the evaluation method, the use of pneumatic pressure test to measure the pressure of the body surface, draw comfortable pressure values in different condition. For weak parts under pressure to the organizational structure of fabrics of the special design, so as to better improve sports bra pressure comfort.

Swimming is a kind of popular sport in the women's movement project. The key in Swimsuit design including no bearing, no bound feeling of comfort and so on. The design of the swimsuit is mainly include the chest pressure comfort design. Zhang Xiaoxu through the use of a variety of experimental equipment to measure fabric each attribute value, choosing the appropriate material, make sample. The following Figure 3, Figure 4. Then use AMI3037-10 air contact pressure tester, pressure measurement and a series of experiment was carried out. Finally will measure the objective pressure combined with subjects of subjective evaluation, the corresponding conclusions. Under the condition of static standing, female one-piece pressure distribution is largely top-down reduced gradually. so The pressure distribution size: shoulder $>$ chest $>$ back $>$ haunch $>$ waist [3]. When designing women's one-piece, therefore, should try to reduce the shoulder, chest pressure, so as to reduce the force binding and pressure on the chest, make it more suitable for women's swimming wear one-piece.

Yoga as a kind of fashion health, attracting more and more women to join. Because of yoga movements, and lasts a long time, so the clothing pressure comfort is particularly important. song xiao xia using the pressure measurement system to conduct stress tests In the clothing people on the test model. then let the experimenter wearing lab-gown under do different yoga movements within a prescribed time period, record the pressure value of each measurement point. Mainly aimed at the prominent part of chest and waist. Measuring figure as follows. 


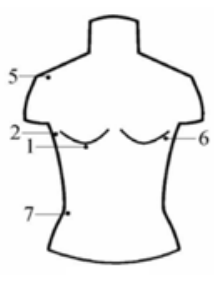

Figure 1. Front.

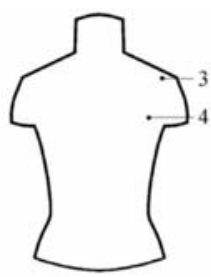

Figure 2. Back.

By having mannequin measured value compared with the experimenters measured pressure value, it is concluded that the static, yoga clothing pressure distribution more uniform, each place little deviation. For different sports, pressure occur great changes and trends is not the same [4]. Comprehensive analysis of pressure on the each parts, It is concluded that in the process of doing yoga exercise, the chest more stress, So it is focus on improving the pressure comfort of chest in the bra design.

In low intensity exercise, researchers mainly through pressure on the chest and the surrounding areas of measurement, it is concluded that the different movement, the pressure on the same area size is different, so the design of sports bra also need to meet the needs of the corresponding movement, to increase the pressure comfort of sports bra.

\subsection{Medium Intensity Exercise}

Sports bras good pressure comfort not only improve human body function, but also to play exercise levels and protect the breasts from damage. Be based on the medium intensity exercise (skiing, walking, boating, aerobic pedal and so on) the pressure comfort of each part of the female body movement is different, and $\mathrm{Wu}$ Zhi Ming study the fabric pressure comfort performance. It is concluded that when the fabric has good elasticity, suitable for use in large area under pressure. Moderate intensity exercise, according to the requirement of the human body each part of pressure comfort to partition design of sports bra, so as to improve the pressure comfort of sports bra [5].

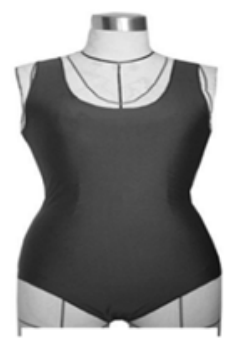

Figure 3. Front.

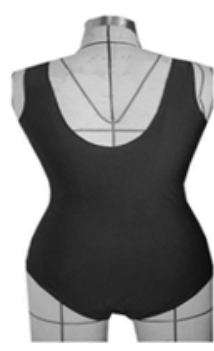

Figure 4. Back.

Jogging is a healthy way of aerobic exercise. Ren jing ping Collect breast movement by using the American Motion Analysis, infrared Motion capture system. In women's bare chest and wear sports bras, the measurement of breast motion amplitude on Each point in the process of walking $(4 \mathrm{~km} / \mathrm{h}$ and $6 \mathrm{~km} / \mathrm{h})$ and running $(8 \mathrm{~km} / \mathrm{h}$ and $10 \mathrm{~km} / \mathrm{h})$ [6]. As can be seen from the results, when the greater the amplitude, the more pressure of the chest. Women in the process of movement, if wear not the right sports bra, chest shake extent will increase, it is easy to hurt breast elastic fiber tissue, causing chest pain. So women doing jogging also should to choose the right sports bra to protect the chest.

For medium intensity exercise, due to the intensity of ascension, the vibration of the movement of chest degree increases in the movement process, So the pursuit of higher pressure comfort of sports bra. Sports bras according to pressure difference can be partitioned design, to improve the pressure comfort.

\subsection{High Intensity Exercise}

The vertical jump movement belongs to the high intensity of movement. Lu ming yan using VICON kinematics analysis system concluded that the vertical jump pressure at various points in the process of dynamic change By capturing the infrared light, data conversion, processing process, etc [7]. It can find that movement in the process of sports bra breast pressure greatly increased and each point pressure more concentrated. She using the German Zebirs Run the gait analysis at the same time finished women in bare-breasted, wear a normal bra, wear sports bras pressure of each measurement point in the process of running. Concludes that each point showed regular fluctuations. compared with the vertical jump movement, the pressure of each point is greatly different. Compared to two kinds of sports, it is concluded that the parts of the body due to the expansion of the skin has a certain amount of comfort in different sports, so the sports bra elastic limit must be considering the amount of the comfortable size, otherwise the body will felling unwell due to compression.

Basketball belongs to endurance sports with High intensity and long duration. Women in the process of movement, the pressure comfort of sports bra for the function of the movement of athletes and physical quality has very important influence. Yan Yu xiu has done related research about that Basketball sports bra pressure effects on heart rate variation index. For sports bras pressure measuring her adopt imported 
FlexiForce201 type thin film pressure sensor and MFF series more film pressure test system which Collect data once every 0.1 s. For HRV measurement using the Polar RS800CX heart rate table that 1 time per $0.6 \mathrm{~s}$ acquisition and use the software of PolarProTrainer5 for statistical analysis. Through statistical subjects pressure and heart rate variation index data eliminate the error, retain valid data. then calculate the average value In each test point pressure and the analysis of the index of heart rate variation trends With the change of pressure value. It is showed that in the process of playing basketball, sports bras clothing pressure have significant influence on 11 indexes. In order to keep the physiological state of the athletes in smooth and comfortable, female chest compression should be controlled in the range of $2.067 \sim$ $3.268 \mathrm{kpa}$ [8]. So in the design of basketball sports bra, women should have certain pressure in chest.

$\mathrm{Xu}$ Jun studied women's beach volleyball clothing pressure comfort by using the Pressure measurement system of LABVIEW virtual instrument. The experimenter dress in particular clothes and put sensors in the different parts of the body Then do different actions for pressure measurement. Then according to the results, the design and improvement of clothing [9]. Contrast before and after experimental data it shows that the improved clothes meet the pressure of comfort in the main parts. The pressure of each place and no more than $2.94 \times 102 \sim 2.94 \times 102$ pa accord with human body physiological requirements.

Artistic gymnastics is accompanied by the beat of music, complete complex, continuous, high strength movement confrontational strong project. The body of different size, different parts of the body and different motion state of each part of the clothing pressure comfort requirements are also different. He wenjuan studied the women's gymnastics in the body of clothing pressure [10]. She uses the Japanese AMI - 3037 pressure test for measuring points. Comprehensive subjective pressure comfort evaluation result, by using the method of parameter interval estimation to build clothing comfortable and clothing pressure of regression model. Through the comparison and analysis data, in the different actions, chest pressure value is also different. The pressure of the chest is big in Play kick, After the kick, Suction kick. But when jumping jacks, the pressure of the chest is smaller. In order to keep the clothing pressure comfort, in view of the special parts, should adopt flexible performance, restorative, scalability good fabrics, thus improve its chest pressure comfort.

For high intensity exercise, the female should attach great importance to the protection of the breast, especially the chest big woman. For the requirement of sports bra, not only to have the effect of reasonable constraints on the chest, but also to meet the requirements of comfort. Researchers in-depth research on the different aspects on the special parts, pressure value range, elastic fabric and so on, in order to improve the pressure comfort of sports bra.

\section{Conclusion}

Sports bras pressure comfort is an important focus in the current research on social, but now research mostly stay in the qualitative, practical applications is not strong. Effects on the pressure comfort of sports bras are various, it will be of human body engineering, structural mechanics, psychology, physiology and so on. So when doing research, should be taken into overall consideration. In addition, still need to combine fabrics, human skin and soft tissue of the relationship between the microstructure and macro properties of light, from molecular and nano structure in-depth exploration, improve sports bra comfort.

\section{References}

[1] Ding Xiaojun, He Ying, Tang Jiefang. The innovation of the clothing pressure comfort experiment design [J]. Journal of zhejiang university of technology, 2013, 30 (1): 55- 58.

[2] Yu Shijia. Women's seamless underwear pressure comfortableness research and its size optimization [D]. 2009: 8- 26.

[3] Zhang Xiaoxu, Mu Hong. Female one-piece comfortable study pressure range $[\mathrm{J}]$. Modern science and technology of silk, 2012, 27 (1): 5- 7.

[4] Song Xiaoxia, Dong Baoyun, Feng Xunwei. Yoga coat pressure objective measurement and analysis [J]. Journal of donghua university, 2010, 36 (5): 506- 510.

[5] Wu Zhiming, Wang Meili. Sports bras partition design based on comfort [J]. Textile journals, 2010, 31 (4).

[6] Ren Jingping, Yan Ying, Zhou Xinglong. Walking and running speed of different sports sports bra breast amplitude [J]. Journal of capital institute of physical education,2015, 2: 172- 177.

[7] Lu Mingyan. The movement of sports bra comfort research and design [D]. soochow university, 2015, 11-39.

[8] Yan Yuxiu, Gao Jie, Jin Zimin, Tang Jiefang, Tao Jianwei. Women's basketball sports bra pressure effects on heart rate variation index [J]. Textile journals, 2014, 35 (6): 100-104.

[9] Xu Jun, Zhang Li, Zhang Feng. Based on the women's beach volleyball clothing pressure comfort of sports biomechanics research [J]. Journal of tianjin university of technology, 2009, 28 (1): 23- 26.

[10] He Wenjuan. Seamless gymnastics women's clothing pressure comfort research and size optimization [D]. Zhejiang Sci-Tech University, 2011.

[11] Xu Jun, Zhou Qing. Sports bra pressure distribution of the subjective evaluation [J]. Textile journals, 2005, 26 (2): 7781 .

[12] Chang Lixia, Zhang Xin. Female fitness and sports bras [J]. Shaanxi textile, 2006: 24-25.

[13] Huang Xiaoqin. Bra cup dart quantity distribution of breast pressure comfort and shape the impact study [D]. donghua university, 2010.

[14] Qian Wei. It's best to wear underwear when motion [J]. The public health, 2013 (4).

[15] Meng Xiangling, Zhang Weiyuan. The research progress of clothing pressure comfort $[\mathrm{J}]$. Textile journals, 2006, 27 (7): $110-112$. 